\title{
Is CD163 - A Marker of Progression in Osteoarthritis?
}

\author{
Keykhosro Mardanpour (iD) ${ }^{1}$, Mahtab Rahbar (iD) ${ }^{2,{ }^{*}}$ and Nyosha Mardanpour ${ }^{3}$ \\ ${ }^{1}$ Orthopedic Department, Kermanshah University of Medical Sciences, Kermanshah, Iran \\ ${ }^{2}$ Pathology Department, Iran University of Medical Sciences, Tehran, Iran \\ ${ }^{3}$ Molecular Research Department, Iran University of Medical Sciences, Tehran, Iran \\ *Corresponding author: Pathology Department, Iran University of Medical Sciences, Tehran, Iran. Tel: +98-9125214011, Email: rahbarahbar@gmail.com
}

Received 2018 May 05; Revised 2018 September 02; Accepted 2018 September 04.

\begin{abstract}
Background: CD163 is a cell surface glycoprotein as known as a hemoglobin/haptoglobin scavenger receptor. The Soluble form of CD163 attenuates macrophages immune response.

Objectives: The aim of this study is to describe the effect of $\mathrm{CD} 163$ phagocytes on progression and joint destruction by osteoarthritis (OA).

Methods: Twenty OA patients participated in this study. The mean age of patients was $68 \pm 8$ years (range 46 to 75 years) and the male to female le ratio was 1.5 , with $10.6 \pm 6.3$ years mean duration of OA disease. Twenty synovial biopsies of OA patients and 10 healthy control (HC) specimens were analyzed for CD163 expression (using the immunohistochemistry method) with soluble CD163 (using the ELISA method) in synovial fluid (SF) as well as SCD163 in the serum of.

Results: The score of CD163 expression in the synovial intima $(\mathrm{P}<0.01)$, the subintima $(\mathrm{P}<0.01)$ and periarticular area $(\mathrm{P}<0.02)$ increased in OA as compared with the healthy control group. Synovial fluid SCD163 and serum sCD163 levels increased in the OA sample population as compared with the healthy control group. Also, Synovial fluid and serum sCD163 positively associated with ESR and CRP $(\mathrm{P}<0.02, \mathrm{P}<0.01$ and $\mathrm{P}<0.06, \mathrm{P}<0.06$ respectively). The mean concentration of SF sCD163 was about 2 times higher than in serum in OA patients $(\mathrm{P}<0.01)$. There was no correlation between clinical findings of patients and SF leukocyte count the $(\mathrm{P}<0.09)$.

Conclusions: This study shows increased numbers of CD163 macrophages with an enhanced phagocytes infiltration within the degenerated synovium as well as the local production of serum and SF SCD163. Therefore, these cells could provide a future therapeutic strategy for osteoarthritis.
\end{abstract}

Keywords: Osteoarthritis, Synovium, CD163 Macrophage, Soluble CD163

\section{Background}

Osteoarthritis (OA) is the most common type of joint disordered which is called degenerative joint disease (DJD) (1). DJD is a kind of disease of the articular surface which is associated with progressive degeneration of the articular surface, or also synovial layer, relative ligaments, and the subchondral areas. The incidence and prevalence of DJD vary through of the world and between different populations. Based on previous studies, about 10 percent of men and 18 percent of women more than 60 years old are involved by degenerative joint disease (1). Synovial inflammation enables the remodeling and repairing of affected tissues. However, the causes of OA are unclear, but it is clarified that OA sometimes started with articular surface damage because of mechanical injuries and accumulation of inflammatory mediators which are secreted from synovial cells into the damaged cartilage sur- face and then lead to cartilage metabolism defects. After the joint injury, chondrocytes attempt to repair articular surface with increases of the production of proteoglycans and collagen and connective tissue around of joint. However, during this period, some enzymes released of inflammatory cells that begin to degrade cartilage of articular surface. Inflammatory mediators originated from inflammatory cells degrade the chondrocytes and synovial lining cells lead to breaking down the articular cartilage lining. The articular surface and all surrounded tissues are affected by OA. During this degrading process, the synovium becomes inflamed and fibrotic. Synovial fluid (SF) accumulated in articular synovial space. This fluid is characterized by less viscosity in greater volume. Also, periarticular tissues including relative tendons as well as ligaments are in stress. The joints become stiff and less mobile. CD163 is a hemoglobin/haptoglobin 
scavenger receptor family (2). The CD163 is expressed on cytoplasm membrane of monocytes and macrophages. Some previous studies reported that the high expression on existent monocytes and macrophages in meanwhile of advanced stages of OA $(3,4)$. The CD163 expression is increased by inflammatory mediators such as glucocorticoids, IL-10, IL-6, and finally the macrophage colony-stimulating factor; it is down-regulated by TNF $\alpha$, lipopolysaccharide (LPS), and interferon- $\gamma$ (IFN $\gamma)(2,5)$. However, CD163 biomarker has been reacted during the inflammatory process by about three different pathways, 1In vitro, The CD163 can be secreted of the cell membrane by metalloproteinase enzyme in response to lipopolysaccharides and becomes soluble CD163 (sCD163). However ,the CD163 (sCD163) has been circulated in plasma (4-6). The sCD163 considers decreasing activation and proliferation of some inflammatory function in vitro (7, 8). 2-CD163 added to a kind of protein tyrosine kinase and casein kinase II-dependent signal that leads to mobilization of calcium, inositol triphosphate production, and some pro-inflammatory mediators such as IL-1 $\beta$, IL6 , and granulocyte-macrophage colony-stimulating factor $(9,10)$. 3-CD163 is a hemoglobin/haptoglobin scavenger receptor complexes, with the highest functional affinity for hemoglobin/haptoglobin complexes (11). However, haptoglobin dependent modulation of oxidative stress molecules, prostaglandin synthesis, and angiogenesis started by the inflammatory process $(12,13)$.

\section{Objectives}

We tried to show that CD163 macrophages could regulate synovial inflammation in OA by controlling of local production of SCD163 in SF and serum possibly. Also, we analyzed CD163 expression on the destructive articular tissue by using the immunohistochemistry method as well as soluble CD163 expression in synovial fluid by using the ELISA method.

\section{Methods}

In this retrospective study, twenty synovial tissue specimens were obtained from advanced OA patients during joint replacement surgery together with synovial fluid aspiration and blood sample simultaneously at Imam Reza and Taleghani hospitals of Kermanshah Medical University between 2012 and 2015. Also, normal human synovium (10 samples) was obtained during orthopedic procedures (such as joint trauma fracture specimen) and their normality confirmed histologically. The demographic and clinical characteristics of 20 OA patients have been shown in Table 1. Five human palatine tonsils as a positive control for CD163 macrophages were obtained from patients undergoing routine tonsillectomy.

\begin{tabular}{lc}
\hline \multicolumn{1}{l}{ Table 1. Demographic and Clinical Characteristics of OA Patients ${ }^{\mathrm{a}}$} \\
\hline \multicolumn{1}{c}{ Parameters } \\
\hline Age, $\mathbf{y}$ & $68 \pm 8$ \\
\hline Male/Female, No. & $12 / 8$ \\
\hline Disease duration, $\mathbf{y}$ & $10.6 \pm 6.3$ \\
\hline Swollen joint count & $9.2 \pm 3.4$ \\
\hline Crepitus & $8.8 \pm 2.7$ \\
\hline Stiffness & $11.2 \pm 3.1$ \\
\hline Tender joint count & $1.8 \pm 3.3$ \\
\hline Serum CRP, mg/dL & $2.7 \pm 2.3$ \\
\hline ESR, mm/hour & $13 \pm 8$ \\
\hline
\end{tabular}

${ }^{\mathrm{a}}$ values are expressed as mean \pm SD unless otherwise indicated.

\subsection{Immunohistochemistry (IHC)}

Thirty synovial biopsy specimens were embedded in paraffin and stained with hematoxylin and eosin for routine histology studies. All paraffin blocks were cut into 6 $\mu \mathrm{m}$ sections and prepared for IHC. First of all, the paraffinembedded blocks were deparaffinized and added to heatinduced antigen retrieval using EnVision FLEX target retrieval solution at low or high $\mathrm{pH}$ (citrate buffer $\mathrm{pH} 6.1$ and Tris/EDTA, pH 9.0, respectively) at $96^{\circ} \mathrm{C}$ for $15 \mathrm{~min}$ with PT-link (Dako, Carpinteria, CA). Based on the threestep of avidin-biotin complex IHC protocol, IHC has been performed (14). The mouse anti-rat monoclonal CD163 (MCA342R, Serotec Ltd, Oxford, UK, dilution 1:50) and also mouse anti-human monoclonal CD163 (SC-20066, Santa Cruz, USA, dilution 1:50) have been used for the primary antibodies. Non-immune serum was applied as a negative control.

\subsection{Manual and Digital Microscope Acquisition}

A digital microscope was used for the real-time slide which was controlled by remote workstations. Furthermore, two pathologists counted the total number of CD163 cells, each specimen in three compartments in the intima, subintima and periarticular area of synovium, using a Nikon Eclipse 80i microscope (Nikon, Tokyo, Japan). The density of CD163 phagocytes was analyzed by doubleblinded observers. It was the number of positive cells (cytoplasmic brownish colored cells) per unit tissue surface area $\left(\mathrm{mm}^{2}\right)$ to define the density of cells. For statistical 
analysis, we used the logarithm of the mean density of two compartments for each sample. Semi-quantitatively, we determined degrees of CD163 cells infiltration into four groups, as follows: $0=$ nil, $\mathrm{I}=$ mild, $\mathrm{II}=$ moderate and III = severe. CD163 cells were counted in three compartments in each specimen. A total number of CD163 phagocytes were determined by sum of the counts for the three compartments. The average numbers of 0,1-19, $20-49$, and $\geq$ 50 were scored as 0 , I, II, and III respectively. Scores were also rechecked randomly by a second pathologist. Interobserver agreement was found $(\kappa=0.69)$.

\subsection{ELISA for Circulating Soluble CD163}

ELISA is one of the best methods for measuring the levels of sCD163 in serum and spinal fluid. We used Human Soluble CD163 ELISA kit in this study (BD Biosciences, San Jose, CA). This assay employs a two-site sandwich ELISA to quantitate CD163 in samples. An antibody specific for CD163 has been pre-coated onto a microplate. Standards and samples are pipetted into the wells and anyCD163 present is bound by the immobilized antibody. After removing any unbound substances, a biotin-conjugated antibody specific for CD163 is added to the wells. After washing, Streptavidin conjugated Horseradish Peroxidase (HRP) is added to the wells. Following a wash to remove any unbound avidin-enzyme reagent, a substrate solution is added to the wells and color develops in proportion to the amount of CD163 bound in the initial step. The color development is stopped and the intensity of the color is measured at $492 \mathrm{~nm}$ wavelength based on ELISA plate reader (15).

\subsection{Statistical Analysis}

All continuous values were expressed as mean \pm SD and categorical variables were presented as a percentage. Spearman's Rho test was used to find correlation and the Mann-Whitney U test used o find a comparison. Also, the logarithm of the serum sCD163 level or the SF sCD163 was used for parametric tests ( $t$-test for comparison and Pearson's test for correlation). P values were applied for the reference table by using a Chi-square. A critical P value of 0.05 was used for all hypothesis testing. All statistics were calculated with SPSS (version 16.0; SSS Inc, Chicago, II).

\section{Results}

For each of the $20 \mathrm{OA}$ and healthy control (HC) subjects who underwent needle arthroscopy, 30 of the synovial biopsy samples were embedded in paraffin and stained with hematoxylin and eosin for routine histologic assessment. An additional 30 biopsy samples were used for frozen sections and IHC. Stained sections were coded and analyzed by two independent observers, who were blinded as to the diagnosis and clinical data (Figure 1).

The semi-quantitative scores for CD163 macrophages in the synovial membrane of OA patients $(n=20)$ and $\mathrm{HC}(\mathrm{n}$ $=10$ ) in three locations of the specimen are summarized in Table 2.

The score of CD163 expression in the synovial intima (P $<0.01)$, the subintima $(\mathrm{P}<0.01)$ and periarticular area $(\mathrm{P}$ $<0.02$ ) increased in OA as compared to HC (Table 3 ).

The presence of CD163 macrophages in three locations were strongly correlated $(\mathrm{r}=0.650, \mathrm{P}<0.01, \mathrm{P}<0.01, \mathrm{P}<$ 0.02 retrospectively). In $\mathrm{HC}$ synovium, the distributions and numbers of CD163 cells were zero. However, in OA synovium, the staining patterns for CD163 were interestingly different in the synovial intima, subintima and periarticular areas as compared with the HC synovium.

Levels of sCD163 were measured by ELISA in SF, as well as in serum form, from 20 OA patients and $10 \mathrm{HCs}$. The SF sCD163 levels were corrected by calculating the ratio of sCD163 to albumin in order to adjust this dilution factor and correctly reflect local production. The synovial fluid sCD163 and serum sCD163 levels increased in the OA group as compared to the healthy control group. The mean concentration of SCD163 was about 2 times higher in SF than in serum in OA patients (6.7 versus $2.9 \mathrm{mg} / \mathrm{L} ; \mathrm{P}<0.01$ ). There is clearly a direct correlation between SF sCD163 production levels and CD163 expression in synovial tissue $(\mathrm{P}<0.03)$ (Table 4 ).

In OA patients, there is a significant positive correlation between the synovial fluid and serum SCD163 with ESR, $\mathrm{CRP}(\mathrm{P}<0.02, \mathrm{P}<0.01$ and $\mathrm{P}<0.06, \mathrm{P}<0.06$ respectively $)$. There was no correlation between the clinical findings of patients and SF leukocyte count $(\mathrm{P}<0.09)$ (Table 5).

\section{Discussion}

Although osteoarthritis is caused by inflammation, breakdown and eventual loss of the cartilage of the joints and associated with aging, trauma, occupational injury possibly the main pathogenesis of the disease remains unclear. In men, hip joint and in women, knees, and hands joints are the common joints that involved by $\mathrm{OA}$. The early changes begin by degeneration of hyaline cartilage of articular surface with possible cartilage fragmentation which leads to the thinning of cartilage and overgrowth of opposing joint surface. The multiple factors show an active role in this process. The recent studies have reported 

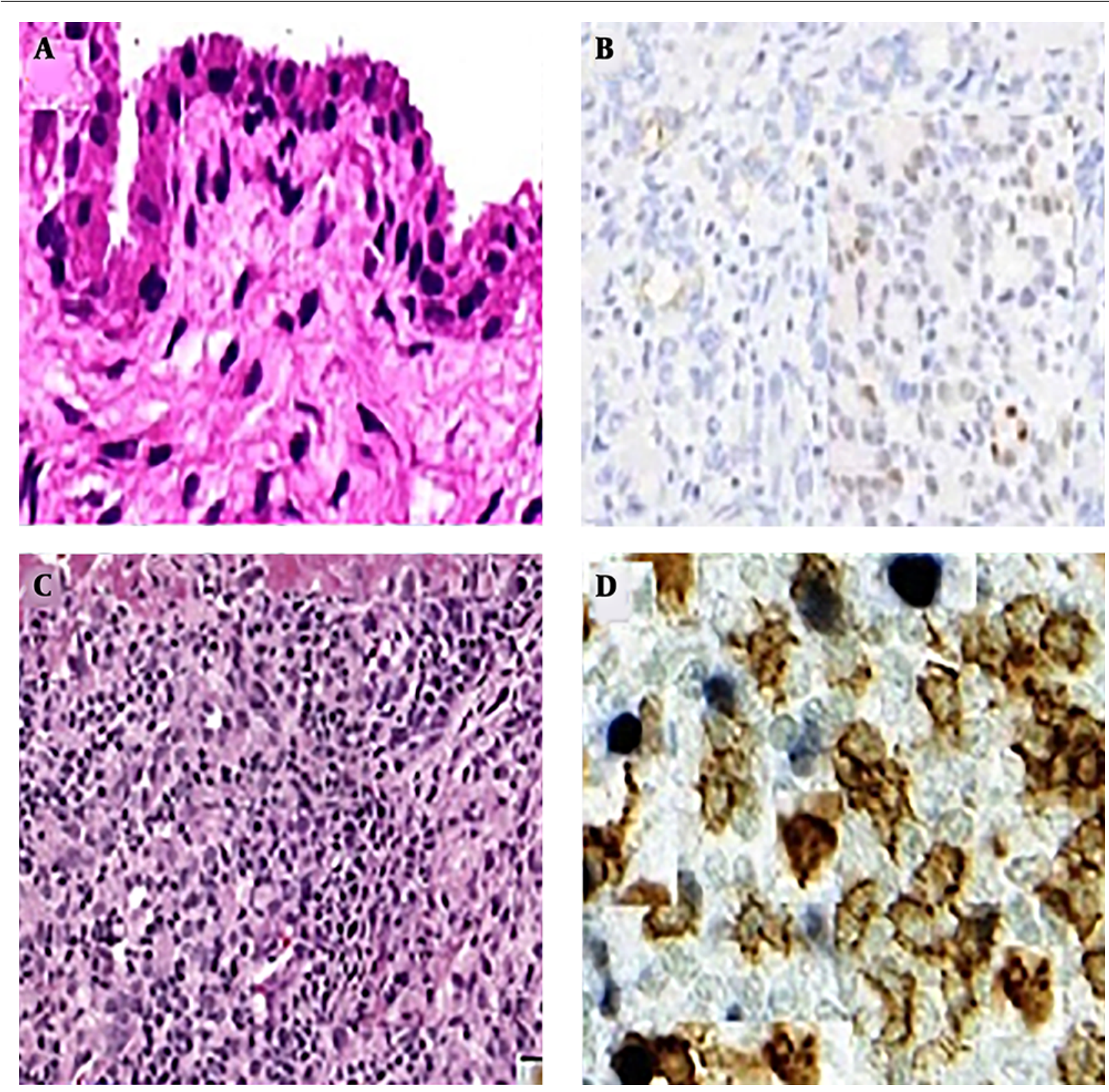

Figure 1. Immunohistochemical staining of CD163 on synovium biopsy (A, B), monoclonal antibody expression of CD163 in healthy control synovium; (C, D), monoclonal antibody expression of CD163 in the synovium of OA patients

the body's immune defense mechanism theory. The role of some of the innate immune factors has been tested in OA models in vivo, including the role of synovial monocytes and macrophages (16). Immunophenotyping markers for the monocytes and macrophages lineage with high sensitivity and specificity have identified (1). The CD163 is considered specific to the phagocytes, with the highest expression on mature macrophages (15). In our study, CD163 levels also appeared to be high in or around vessel walls in synovium and periarticular tissues. Thus, the presence of CD163 macrophages in OA synovium suggests that CD163 could be an immunoregulatory factor in OA disease. Also, in a previous study, soluble CD163 correlated with disease activity markers was reported in early rheumatoid arthritis (17).

Soluble CD163 (sCD163) which comes from of the phagocytes receptors exists in plasma and cerebrospinal fluid. Soluble CD163 is originated from ectodomain shed- 


\begin{tabular}{|c|c|c|c|c|c|c|c|c|c|c|c|c|}
\hline \multirow{2}{*}{ Patient } & \multicolumn{4}{|c|}{ Intima } & \multicolumn{4}{|c|}{ Subintima } & \multicolumn{4}{|c|}{ Periarticular } \\
\hline & $\mathbf{0}$ & I & II & III & o & I & II & III & $\mathbf{0}$ & I & II & III \\
\hline 1 & & & + & & & & + & & & & & + \\
\hline 2 & & & + & & & & & + & & & & + \\
\hline 3 & & + & & & & & + & & & & + & \\
\hline 4 & & + & & & & & + & & & & & + \\
\hline 5 & & & + & & & & + & & & & & + \\
\hline 6 & & & + & & & & + & & & & + & \\
\hline 7 & & & & + & & & & + & & & & + \\
\hline 8 & & & + & & & & + & & & & + & \\
\hline 9 & & & + & & & + & & & & & & + \\
\hline 10 & & & + & & & & + & & & & & + \\
\hline 11 & & + & & & & & + & & & & + & \\
\hline 12 & & & + & & & & + & & & & & + \\
\hline 13 & & + & & & & + & & & & & + & \\
\hline 14 & & & + & & & & + & & & & & + \\
\hline 15 & & + & & & & & + & & & & + & \\
\hline 16 & & & + & & & & + & & & & & + \\
\hline 17 & & & + & & & & + & & & & & + \\
\hline 18 & & & + & & & & + & & & & + & \\
\hline 19 & & + & & & & + & & & & & + & \\
\hline 20 & & & + & & & & + & & & & & + \\
\hline
\end{tabular}

Table 3. Immunohistochemical Evaluation of the CD163 Expression in the Synovial Intima, Subintima and Periarticular Areas of Synovial Specimens in OA Patients

\begin{tabular}{lccc}
\hline CD 163 Expression & Mean Numbers & Score & PValue \\
\hline Intima & $20-49$ & II & $<0.01$ \\
Sub Intima & $20-49$ & II & $<0.01$ \\
Periarticular & $\geq 50$ & III & $<0.02$ \\
\hline
\end{tabular}

Table 4. Correlations Between Serum sCD163 Levels, SF sCD163 and Clinical and Synovium Tissue CD163 Expression in OA Patients

\begin{tabular}{llc}
\hline Parameters & Synovium Tissue & P Value \\
\hline Serum sCD163 & CD163 Expression & $<0.12$ \\
Synovial Fluid sCD163 & CD163 Expression & $<0.03$ \\
\hline
\end{tabular}

ding of the membrane-receptor. Soluble CD163 shedding could be generated of enzymatic cleavage which could be happened in the inflammatory process. Soluble CD163 is expressed in a large group of inflammatory diseases such as cirrhosis, type II diabetic disease, macrophage activation syndrome, Gaucher's disease, sepsis, HIV infection, rheumatoid arthritis, and Hodgkin Lymphoma. It remains to be determined whether sCD163 is an early predictor of OA flare $(1,17)$. The current study was the first to investigate the correlation between the CD163 expression marker on the synovial membrane and periarticular tissues with sCD163 in clinical findings of OA patients. Our study shows that in OA patients the ratio of sCD163 increased and was about 2 times higher in SF than in serum. Also, there is clearly a direct, significant correlation between SF sCD163 production levels with the CD163 expression in synovial tissue, ESR and CRP. In addition, there was an increased percentage of CD163 macrophages with enhanced phagocytes and migratory activities within the OAlike synovium. Possibly, the increased phagocytosis of this cell population within degraded synovium and periarticular tissues may sensitize them to cell death, leading to the secretion of additional inflammatory cytokines, and result in the progressive degradation of cartilage in osteoarthritis. Otherwise, the scavenger function of CD163 phagocytes within subintima and periarticular tissues like articular cartilage $(18,19)$ could potentially facilitate the other role of the CD163 phagocytes in OA. The results of our study were supported by previous studies which showed 


\begin{tabular}{|c|c|c|c|c|}
\hline \multirow{2}{*}{ Parameters } & \multicolumn{2}{|c|}{ S sCD163 } & \multicolumn{2}{|c|}{ SF sCD163 } \\
\hline & $\mathbf{R}$ & PValue & $\mathbf{R}$ & PValue \\
\hline Disease duration & 0.07 & 0.50 & 0.51 & 0.13 \\
\hline Swollen joints & 0.16 & 0.15 & 0.23 & 0.15 \\
\hline Stiffness & 0.09 & 0.80 & 0.20 & 0.10 \\
\hline CRP & 0.26 & 0.06 & 0.48 & 0.01 \\
\hline ESR & 0.26 & 0.06 & 0.52 & 0.02 \\
\hline WBC & 0.16 & 0.15 & 0.05 & 0.90 \\
\hline
\end{tabular}

Abbreviations: CRP, C reactive protein; ESR, erythrocyte sedimentation rate; OA, osteoarthritis; sCD163, soluble CD163; SF, synovial fluid; S sCD163, serum soluble CD163; WBC, white blood cell.

that CD163 biomarker is an endocytic receptor for both the Hemoglobin-Haptoglobin complexes and also bacteria (11, 20).

\subsection{Conclusion}

Plasma and synovial fluid levels of macrophagederived SCD163, and also CD 163 macrophages, reflected the role of this valuable biomarker in disease progression and joint destruction in early OA patients. However, the presence of continued articular stress and damage creates a vicious cycle and this scavenger capability can be overridden. The results could be used for the further therapeutic approach in OA patients.

\section{Acknowledgments}

We would like to extend our thanks to all the respected colleagues who cooperated in this study. Also, the authors thank the Orthopedic Departments of Imam Reza and Taleghani hospitals of Kermanshah University of Medical Sciences for supporting this study.

\section{Footnotes}

Authors' Contribution: Keykhosro Mardanpour designed and directed the study; analyzed and organized the data. Mahtab Rahbar performed collected data and data analysis. Nyosha Mardanpour contributed to the manuscript writing and organized tables.

Ethical Considerations: Approval from our hospitals was obtained prior to initiating the study and all patients gave consent for research. All demographic data were obtained from the Traumatic Center database of three Kermanshah University hospitals and therefore, institutional review board approval is not applicable.

\section{References}

1. Jiao K, Zhang J, Zhang M, Wei Y, Wu Y, Qiu ZY, et al. The identification of CD163 expressing phagocytic chondrocytes in joint cartilage and its novel scavenger role in cartilage degradation. PLoS One. 2013;8(1). e53312. doi: 10.1371/journal.pone.0053312. [PubMed: 23326413]. [PubMed Central: PMC3543453].

2. Hogger P, Dreier J, Droste A, Buck F, Sorg C. Identification of the integral membrane protein RM3/1 on human monocytes as a glucocorticoid-inducible member of the scavenger receptor cysteine-rich family (CD163).J Immunol.1998;161(4):1883-90. [PubMed: 9712057].

3. Sanchez C, Domenech N, Vazquez J, Alonso F, Ezquerra A, Dominguez $\mathrm{J}$. The porcine 2A10 antigen is homologous to human CD163 and related to macrophage differentiation. J Immunol. 1999;162(9):5230-7. [PubMed: 10227997].

4. Sulahian TH, Hogger P, Wahner AE, Wardwell K, Goulding NJ, Sorg C, et al. Human monocytes express CD163, which is upregulated by IL-10 and identical to p155. Cytokine. 2000;12(9):1312-21. doi: 10.1006/cyto.2000.0720. [PubMed: 10975989].

5. Hintz KA, Rassias AJ, Wardwell K, Moss ML, Morganelli PM, Pioli PA, et al. Endotoxin induces rapid metalloproteinase-mediated shedding followed by up-regulation of the monocyte hemoglobin scavenger receptor CD163. J Leukoc Biol. 2002;72(4):711-7. [PubMed: 12377940].

6. Droste A, Sorg C, Hogger P. Shedding of CD163, a novel regulatory mechanism for a member of the scavenger receptor cysteinerich family. Biochem Biophys Res Commun. 1999;256(1):110-3. doi: 10.1006/bbrc.1999.0294. [PubMed: 10066432].

7. Hogger P, Sorg C. Soluble CD163 inhibits phorbol ester-induced lymphocyte proliferation. Biochem Biophys Res Commun. 2001;288(4):8413. doi: 10.1006/bbrc.2001.5845. [PubMed: 11688984].

8. Frings W, Dreier J, Sorg C. Only the soluble form of the scavenger receptor CD163 acts inhibitory on phorbol ester-activated Tlymphocytes, whereas membrane-bound protein has no effect. FEBS Lett. 2002;526(1-3):93-6. [PubMed: 12208511].

9. Van den Heuvel MM, Tensen CP, van As JH, Van den Berg TK, Fluitsma DM, Dijkstra CD, et al. Regulation of CD 163 on human macrophages: Cross-linking of CD163 induces signaling and activation.J Leukoc Biol. 1999;66(5):858-66. [PubMed: 10577520].

10. Ritter M, Buechler C, Kapinsky M, Schmitz G. Interaction of CD163 with the regulatory subunit of casein kinase II (CKII) and dependence of CD163 signaling on CKII and protein kinase C. EurJ Immunol. 2001;31(4):999-1009. doi: 10.1002/1521-4141(200104)31:4<999::AIDIMMU999\&3.0.CO;2-R. [PubMed: 11298324]. 
11. Kristiansen M, Graversen JH, Jacobsen C, Sonne O, Hoffman HJ, Law SK, et al. Identification of the haemoglobin scavenger receptor. Nature. 2001;409(6817):198-201. doi: 10.1038/35051594. [PubMed: 11196644].

12. Langlois MR, Delanghe JR. Biological and clinical significance of hap toglobin polymorphism in humans. Clin Chem. 1996;42(10):1589-600. [PubMed: 8855140].

13. Schaer DJ, Boretti FS, Schoedon G, Schaffner A. Induction of the CD163-dependent haemoglobin uptake by macrophages as a novel anti-inflammatory action of glucocorticoids. $\mathrm{Br}$ J Haematol. 2002;119(1):239-43. [PubMed: 12358930].

14. Baeten D, Demetter P, Cuvelier CA, Kruithof E, Van Damme N, De Vos $\mathrm{M}$, et al. Macrophages expressing the scavenger receptor CD163: link between immune alterations of the gut and synovial inflammation in spondyloarthropathy. J Pathol. 2002;196(3):343-50. doi 10.1002/path.1044. [PubMed: 11857499].

15. Kusi KA, Gyan BA, Goka BQ, Dodoo D, Obeng-Adjei G, Troye-Blomberg $\mathrm{M}$, et al. Levels of soluble CD163 and severity of malaria in children in Ghana. Clin Vaccine Immunol. 2008;15(9):1456-60. doi 10.1128/CVI.00506-07. [PubMed: 18632918]. [PubMed Central: PMC2546665].
16. Orlowsky EW, Kraus VB. The role of innate immunity in osteoarthritis: When our first line of defense goes on the offensive. J Rheumatol. 2015;42(3):363-71. doi: 10.3899/jrheum.140382. [PubMed: 25593231]. [PubMed Central: PMC4465583].

17. Greisen SR, Moller HJ, Stengaard-Pedersen K, Hetland ML, HorslevPetersen K, Junker P, et al. Macrophage activity assessed by soluble CD163 in early rheumatoid arthritis: Association with disease activity but different response patterns to synthetic and biologic DMARDs. Clin Exp Rheumatol. 2015;33(4):498-502. [PubMed: 25962601].

18. Bigg HF, Rowan AD. The inhibition of metalloproteinases as a therapeutic target in rheumatoid arthritis and osteoarthritis. Curr Opin Pharmacol. 2001;1(3):314-20. [PubMed: 11712757].

19. Martel-Pelletier J, Welsch DJ, Pelletier JP. Metalloproteases and inhibitors in arthritic diseases. Best Pract Res Clin Rheumatol. 2001;15(5):805-29. doi:10.1053/berh.2001.0195. [PubMed: 11812023].

20. Fabriek BO, van Bruggen R, Deng DM, Ligtenberg AJ, Nazmi K, Schornagel $\mathrm{K}$, et al. The macrophage scavenger receptor CD163 functions as an innate immune sensor for bacteria. Blood. 2009;113(4):887-92. doi: 10.1182/blood-2008-07-167064. [PubMed: 18849484]. 